\title{
DEGLUTIÇÃO DE RESPIRADORES ORAIS E NASAIS: AVALIAÇÃO CLÍNICA FONOAUDIOLÓGICA E ELETROMIOGRÁFICA
}

\author{
Swallowing of oral and nose breathers: \\ speech-language and electromyography assessment
}

\author{
Tais Regina Hennig (1), Ana Maria Toniolo da Silva (2), Angela Ruviaro Busanelo ${ }^{(3)}$, \\ Flávia Leães de Almeida ${ }^{(4)}$, Luana Cristina Berwig ${ }^{(5)}$, Luane de Moraes Boton ${ }^{(6)}$
}

\section{RESUMO}

Objetivo: avaliar e comparar a deglutição de sujeitos respiradores orais e nasais, por meio da avaliação clínica e eletromiográfica dos músculos orbiculares orais, superior e inferior. Métodos: participaram deste estudo 16 sujeitos na faixa etária de 6:8 a 10:10 distribuídos em dois grupos, um de respiradores orais e outro de respiradores nasais. Foram submetidos à avaliação clínica fonoaudiológica e eletromiográfica de superfície. O exame fonoaudiológico contemplou as estruturas e funções do sistema estomatognático, e a avaliação eletromiográfica consistiu na captação da atividade elétrica dos músculos orbiculares orais, superior e inferior, durante as situações de isometria e de deglutição. Da avaliação clínica consideraram-se somente os resultados referentes à deglutição, observando-se a presença de ação labial e mentual, e de projeção lingual, as quais foram analisados de forma descritiva. Para análise estatística dos dados eletromiográficos, utilizou-se o Teste t para comparação entre os grupos. A significância adotada foi de $5 \%(p<0,05)$. Resultados: na avaliação clínica foram observadas alterações na deglutição, sendo que $87,5 \%$ dos sujeitos respiradores orais apresentaram ação labial, $75 \%$ ação mentual e $75 \%$ projeção lingual como características da deglutição adaptada, enquanto que nenhum dos sujeitos respiradores nasais apresentou tais alterações. Na avaliação eletromiográfica, verificou-se atividade muscular superior nos respiradores orais, em comparação aos respiradores nasais, em ambos os músculos estudados com diferença estatisticamente significante. Conclusão: concluiu-se que os respiradores orais apresentam alterações evidentes na função de deglutição, verificadas por meio da avaliação clínica fonoaudiológica e da avaliação eletromiográfica, quando comparados aos respiradores nasais.

DESCRITORES: Deglutição; Eletromiografia; Músculos Faciais; Respiração Bucal; Sistema Estomatognático

(1) Aluna do Curso de Graduação em Fonoaudiologia da Universidade Federal de Santa Maria, UFSM, Santa Maria, RS.

(2) Fonoaudióloga; Professora Associada do Departamento de Fonoaudiologia da Universidade Federal de Santa Maria, UFSM, Santa Maria, RS; Doutora em Ciências dos Distúrbios da Comunicação Humana pela Universidade Federal de São Paulo.

(3) Fonoaudióloga; Professora Substituta do Curso de Graduação em Fonoaudiologia da Universidade Federal de Santa Maria, UFSM, Santa Maria, RS; Mestre em Distúrbios da Comunicação Humana pela Universidade Federal de Santa Maria.

(4) Fonoaudióloga; Fonoaudióloga do Município de Manoel Viana, RS; Mestranda em Distúrbios da Comunicação Humana pela Universidade Federal de Santa Maria.

(5) Aluna do curso de Graduação em Fonoaudiologia da Universidade Federal de Santa Maria, UFSM, Santa Maria, RS.

\section{INTRODUÇÃO}

A função respiratória é a primeira estabelecida ao nascimento, sendo vital para o organismo e deve ocorrer, preferencialmente, por via nasal para que o ar seja purificado, filtrado e aquecido antes de chegar aos pulmões e assim, proteger as vias aéreas superiores e favorecer a oxigenação. Além disso, a respiração nasal caracteriza-se como fundamen-

\footnotetext{
(6) Fonoaudióloga da Associação de Pais e Amigos dos Excepcionais, APAE, Cacequi, RS; Mestranda em Distúrbios da Comunicação Humana pela Universidade Federal de Santa Maria.
}

Conflito de interesses: inexistente 
tal para o crescimento e desenvolvimento adequados do complexo craniofacial do indivíduo e o bom funcionamento das demais funções estomatognáticas ${ }^{1-5}$

O indivíduo que apresentar algum impedimento neste processo adotará o padrão oral ou oronasal de respiração, podendo ter causa orgânica ou nãoorgânica (este último também denominado funcional ou vicioso). Quando orgânico, pode ter como causas hipertrofia das tonsilas, faríngea e/ou palatina, desvio de septo, corpo estranho, tumores, pólipos, fraturas, atresias, conchas nasais hipertróficas, rinite crônica, entre outros. Já a respiração viciosa, poderá ocorrer por resfriados, hábitos orais, resultante de fatores orgânicos reparados, ou ainda por flacidez dos músculos faciais e mastigatórios ${ }^{6}$.

Alterações craniofaciais e dentárias, dos órgãos fonoarticulatórios e das funções orais, e, em alguns casos, alterações corporais são características comumente encontradas na respiração oral 5-12. Dentre as funções orais que poderão estar alteradas, tem-se a deglutição, uma vez que estes pacientes tendem a apresentar flacidez e alteração de posição habitual das estruturas orofaciais devido ao uso inadequado das mesmas ${ }^{9,10,12-15}$.

Sendo assim, estas alterações poderão determinar a ocorrência de anormalidades na deglutição como interposição lingual, presença de contração da musculatura periorbicular, diminuição da contração do masseter, contração do músculo mentual, interposição de lábio inferior, movimento de cabeça, ruídos e presença de resíduos alimentares após a deglutição ${ }^{9,16}$.

A avaliação das estruturas e funções do sistema estomatognático (SE), inclusive da deglutição, ainda é realizada de forma subjetiva. Entretanto, os avanços e estudos científicos têm possibilitado complementar a avaliação clínica por meio de exames objetivos, como a eletromiografia (EMG) de superfície. A EMG estuda os sinais elétricos emanados pelos músculos no momento da contração muscular ${ }^{17}$, auxiliando na avaliação, no diagnóstico e no tratamento fonoaudiológico de forma fidedigna ${ }^{18-20}$.

Assim, este trabalho justifica-se pela importância da relação entre dados subjetivos e objetivos, e propõe-se avaliar e comparar a deglutição de sujeitos respiradores orais e nasais por meio da avaliação clínica fonoaudiológica e eletromiográfica dos músculos orbiculares orais, superior e inferior.

\section{MÉTODOS}

Esse trabalho apresenta caráter observacional, transversal e retrospectivo, sendo realizado com base no banco de dados de um projeto de pesquisa desenvolvido no Laboratório de Motricidade Oral do Serviço de Atendimento Fonoaudiológico (SAF) da instituição de origem.

Para compor a amostra do estudo, foram triados 306 sujeitos de primeira a quinta série do ensino fundamental de escolas públicas do município de Santa Maria - RS, os quais foram separados em grupo de estudo (GE) e grupo de controle (GC), conforme os critérios de inclusão e de exclusão.

Os sujeitos deveriam ter, obrigatoriamente, idade entre 6 e 11 anos, além de respeitar os seguintes critérios de inclusão: respiração oral viciosa para compor o GE; e ausência de respiração mista ou predominantemente oral para o GC. Como critérios de exclusão, ambos os grupos não poderiam ter recebido tratamento fonoaudiológico e apresentar sinais evidentes de comprometimento neurológico; e, exclusivamente para o GE, apresentar alteração oclusal significativa que não permitisse o vedamento labial espontâneo.

Dos 306 sujeitos avaliados inicialmente, 151 não se adequaram aos critérios da pesquisa, 96 atenderam aos critérios do GC e 59 aos critérios do GE. Apenas os responsáveis de 36 sujeitos interessaram-se e autorizaram a participação no estudo.

Os sujeitos selecionados realizaram avaliação fonoaudiológica, ortodôntica e otorrinolaringológica, para a comprovação da adequação efetiva dos critérios pré-estabelecidos. Após essas avaliações, 16 sujeitos alcançaram a etapa final do estudo, oito no GE e oito no GC, ambos os grupos com cinco sujeitos do sexo feminino e três do masculino, na faixa etária de 6:8 - 10:10. Com a finalidade de atingir os objetivos iniciais, os mesmos foram então submetidos à avaliação clínica fonoaudiológica e eletromiográfica.

A avaliação fonoaudiológica contemplou o exame do SE - estruturas e funções, no que diz respeito à morfologia extra (lábios, bochechas, mandíbula, crânio/face e ATM) e intra-oral (língua e palato duro e mole), e as funções de sucção, mastigação, deglutição, respiração e fala. Desta etapa, foram analisadas exclusivamente as variáveis: ação labial, ação mentual e a projeção lingual durante a deglutição, por caracterizarem a função como alterada.

A avaliação eletromiográfica consistiu na captação da atividade elétrica dos músculos orbiculares orais, superior (OS) e inferior (OI), durante as situações de isometria e de deglutição. A isometria (contração voluntária máxima - CVM) foi realizada para posterior normalização dos resultados e adotou-se como padrão para tal, a compressão recíproca dos lábios com dentes ocluídos por 5 segundos para evitar a fadiga muscular ${ }^{21}$. A deglutição foi avaliada durante a ingesta de quatro goles seriados 
de líquido (água), durante aproximadamente 15 segundos, realizados sob comando ${ }^{22}$.

Durante as coletas, os sujeitos permaneceram sentados, em posição confortável, orientados pelo Plano Frankfurt e com olhos abertos, sendo realizadas pelo menos três coletas para cada um dos testes em busca do melhor sinal eletromiográfico ${ }^{23,24}$.

Segundo a padronização internacional ${ }^{24}$, para a captação do sinal eletromiográfico, foram utilizados pré-amplificadores ativos com entrada diferencial (Lynx Tecnologia Eletrônica Itda.), ligados a eletrodos de $\mathrm{Ag} / \mathrm{AgCl}$ do tipo DOUBLE (Hal Indústria e Comércio Itda.). Os eletrodos possuíam formato de disco, distância fixa de $20 \mathrm{~mm}$ entre os mesmos, 10 $\mathrm{mm}$ de diâmetro e $2 \mathrm{~mm}$ de superfície de contato, gel condutor em quantidade fixa e estabelecida pelo fabricante, ganho de 20X, impedância de entrada de $10 \mathrm{G} \Omega$ e taxa de rejeição de modo comum de $>100 \mathrm{~dB}$. A colocação dos eletrodos baseou-se no proposto pela literatura ${ }^{17,24} \mathrm{e}$, para evitar interferências eletromiográficas, foi colocado um eletrodo de referência na testa do sujeito. Para diminuir a interferência da impedância oferecida pela pele ${ }^{24}$, a mesma foi previamente preparada realizando-se a limpeza nos locais de colocação dos eletrodos com álcool etílico $70 \%$ e algodão. O local das coletas ${ }^{24}$ teve o chão revestido por material emborrachado e tomou-se o cuidado de distanciar e desligar equipamentos que pudessem interferir eletromagneticamente no exame.

Os sinais eletromiográficos foram condicionados e amplificados utilizando o equipamento EMG 1200 (Lynx Tecnologia Itda.), com oito canais de entrada, conversor $A / D$ de 16 bits e faixa de entrada de $+/-2$ V. Foi utilizado filtro do tipo Butterworth com frequência de corte passa alto de $10 \mathrm{~Hz}$ e passa baixo $1000 \mathrm{~Hz}$. Os sinais foram coletados pelo Software Biolnspector 1.8. (Lynx), quantificados em rootmean-square (RMS), expressos em $\mu \mathrm{V}$ (microvolts) e salvos em computador portátil sem conexão com a rede elétrica.

Após levantamento dos dados eletromiográficos, foi realizada a escolha qualitativa do melhor sinal, quando se considerou o sinal com menor interferência de ruído, para posterior análise quantitativa ${ }^{24}$. Para a análise quantitativa dos mesmos, o sinal foi processado em RMS através do Software AqDAnalysis 7.0 (Lynx).

Em virtude da sensibilidade do sinal eletromiográfico aos fatores intrínsecos e extrínsecos, a normalização do sinal eletromiográfico foi necessária para possibilitar a comparação adequada entre sujeitos, músculos e aquisições ${ }^{17,20,23-25}$.

A técnica de normalização remeteu à transformação dos valores absolutos da amplitude $(\mu \mathrm{V})$ em valores relativos, referentes a um valor de amplitude caracterizado como 100\%. Este estudo calculou os valores normalizados com base na média da CVM dos músculos orbiculares superior e inferior das crianças respiradoras nasais ${ }^{24}$.

O presente estudo foi registrado e aprovado no Comitê de Ética em Pesquisa da instituição de origem sob o $n^{\circ}$ 0067.0.243.000-06.

Os dados clínicos foram analisados de forma descritiva e para análise estatística dos dados eletromiográficos, foi realizado o teste de normalidade Shapiro-Wilks, sendo aplicado, para comparação do GE com o GC, o Teste t. A significância adotada foi de $5 \%(p<0,05)$.

\section{RESULTADOS}

Os resultados das avaliações clínica fonoaudiológica e eletromiográfica dos músculos orbiculares orais, superior e inferior, durante a deglutição são apresentados na Tabela 1 e Tabela 2, respectivamente.

\section{DISCUSSÃO}

Como resultados da avaliação clínica fonoaudiológica da deglutição, observou-se que nenhum dos oito sujeitos respiradores nasais apresentou alterações na deglutição, considerando-se interposição lingual, presença de contração da musculatura periorbicular, diminuição da contração do masseter, contração do músculo mentual, interposição de lábio inferior, movimento de cabeça, ruídos e presença de resíduos alimentares após a deglutição. Quanto aos sujeitos respiradores orais, sete apresentaram ação labial, seis tiveram ação mentual e seis projeção lingual (Tabela 1).

$\mathrm{Na}$ avaliação eletromiográfica dos músculos orbiculares orais durante a deglutição (Tabela 2), verificou-se atividade muscular superior nos respiradores orais em comparação aos respiradores nasais, em ambos os músculos estudados, com diferença estatisticamente significante $(p<0,05)$.

$\mathrm{Na}$ respiração oral, em virtude das inúmeras alterações estruturais, ocorrem também alterações funcionais, como a deglutição adaptada, e esta pode ser caracterizada pela ação labial, ação mentual e projeção lingual ${ }^{9,10,12-15}$.

A projeção lingual ocorre devido à diminuição de tônus e à postura rebaixada de língua, evidenciadas no respirador oral. Na tentativa de corrigir estas alterações, a musculatura perioral, incluindo músculos orbiculares orais e mentual, atuará de forma mais ativa objetivando restabelecer o selamento labial necessário para a adequação da respiração ${ }^{9,12,15,25}$. 
Tabela 1 - Distribuição dos valores absolutos e relativos quanto às variáveis consideradas na avaliação clínica fonoaudiológica da deglutição no grupo estudo $(n=8)$

\begin{tabular}{llc}
\hline Variáveis & RO & RO \\
\hline Ação labial & 7 & 87,5 \\
Ação mentual & 6 & 75 \\
Projeção lingual & 6 & 75 \\
\hline
\end{tabular}

Legenda: $\mathrm{RO}$ - respiradores orais viciosos.

Tabela 2 - Médias da atividade muscular dos orbiculares orais, superior e inferior, durante a deglutição nos sujeitos respiradores orais viciosos e nasais

\begin{tabular}{|c|c|c|c|c|}
\hline & & $\begin{array}{c}\text { RN } \\
\text { Média (DP) } \\
\end{array}$ & $\begin{array}{c}\text { RO } \\
\text { Média (DP) } \\
\end{array}$ & $p$ \\
\hline \multirow{2}{*}{ Deglutição } & OS & $8,24(6,28)$ & $23,83(12,12)$ & $0,00608^{\star}$ \\
\hline & OI & $8,64(5,59)$ & $34,44(17,08)$ & $0,00117^{*}$ \\
\hline
\end{tabular}

Legenda: RN - respiradores nasais, RO - respiradores orais viciosos, DP - desvio padrão, OS - músculo orbicular superior, OI músculo orbicular inferior.

* Significância pelo Teste t $(p<0,05)$

Verificou-se, concordando com a literatura ${ }^{9}$, que a maioria dos sujeitos com respiração oral deste estudo apresentou ação labial, ação mentual e projeção lingual durante a deglutição, caracterizando a função como alterada, o que não foi observado nos respiradores nasais.

A participação compensatória da musculatura perioral, incluindo músculos orbiculares orais e mentual, durante a deglutição representa uma forma de adaptação do SE às alterações morfológicas decorrentes da respiração oral, uma vez que a musculatura mastigatória não desempenha a atividade muscular necessária para realização desta função ${ }^{13,25,26}$.

Além das alterações clínicas da deglutição observadas neste estudo, também foram encontradas alterações na avaliação eletromiográfica desta função. Por meio desta avaliação objetiva, verificouse maior atividade dos músculos orbiculares orais nos sujeitos respiradores orais quando comparados aos respiradores nasais, inclusive com diferença estatisticamente significante, o que vai ao encontro dos achados da literatura ${ }^{27}$.

A atividade elétrica aumentada dessa musculatura, nos respiradores orais, é confirmada por estudos que mostram que a atividade dos orbiculares orais é maior, inclusive no repouso, objetivando manter o contato labial, uma vez que o contato entre os lábios é fundamental para que esta função ocorra ${ }^{28}$.

A maior atividade elétrica dos músculos avaliados nos respiradores orais, quando comparados com os respiradores nasais, sugere que, nestes sujeitos, esta musculatura desempenha maior esforço na realização do selamento labial.

O maior esforço para manter o selamento labial relaciona-se com a postura separada dos mesmos, a qual os respiradores orais estão habituados. É decorrente, também, da hipotonicidade labial, encurtamento do lábio superior e eversão do lábio inferior, determinados pela hipofuncionalidade de ambos os lábios nesses sujeitos para permitir a passagem de ar pela boca $8,9,11,12,15,27,29$. A literatura relata que a atividade elétrica dos músculos avaliados está mais relacionada com a competência labial do que com a existência de má oclusão ${ }^{19}$.

Comparando-se a atividade muscular entre os músculos orbiculares orais, observou-se, neste estudo, que o orbicular inferior foi mais ativo do que o correspondente superior, desempenhando maior atividade muscular durante a deglutição nos sujeitos com respiração oral. Este aumento da atividade muscular do orbicular inferior pode ocorrer devido à atuação conjunta do músculo mentual, objetivando compensar a hipofuncionalidade verificada nos respiradores orais ${ }^{26}$.

Apesar da discordância sobre a predominância do músculo orbicular inferior sobre o superior durante a deglutição de respiradores orais ${ }^{29}$, sabese que nas demais funções do SE também pode haver, assim como na deglutição, padrão mais ativo no orbicular inferior, visto que a atividade muscular nessas funções seria semelhante, já que utilizam as mesmas estruturas ${ }^{18}$.

Deste modo, o desequilíbrio anatômico e funcional existente nos respiradores orais determina uma 
série de alterações durante a função de deglutição, verificadas por meio da avaliação clínica fonoaudiológica, além do padrão alterado de atividade muscular dos músculos orbiculares orais, superior e inferior, por meio da EMG.

\section{CONCLUSÃO}

A partir desse estudo, conclui-se que:

- Na avaliação clínica a maioria dos sujeitos res- piradores orais apresentou alteração da deglutição com presença de ação labial, ação mentual e projeção lingual enquanto que os respiradores nasais não apresentaram nenhuma dessas alterações.

- Na avaliação eletromiográfica a atividade muscular nos respiradores orais foi superior em comparação aos respiradores nasais, em ambos os músculos estudados, com diferença estatisticamente significante.

\begin{abstract}
Purpose: to evaluate and compare the swallowing of mouth and nose breathers through of speechlanguage and electromyography evaluation concerning the orbicularis oris muscles, and lower and upper lips. Methods: 16 subjects aged 6:8 - 10:10 and divided on two groups participated in this study, one with mouth and another one with nose breathers. Both groups were submitted to clinical and surface electromyography evaluation. The speech-language evaluation contemplated the exam of the structures and functions of the stomatognathic system. The electromyography evaluation consisted of the captivation of the electrical activity at the orbicularis oris muscles, and lower and upper lips, during isometry and swallowing situations. In the clinical results were considered only the swallowing variables. Therefore, our study could observe the presence of labial and mentalis action and tongue projection, that were analyzed in a descriptive form. The statistical analysis of the electromyography results was done through the $t$ test in order to compare the groups. The statistical considered significance was $5 \%(p<0.05)$. Results: in the clinical evaluation it was verified that any of the nose breathers showed atypical swallowing characteristics while $7(87.5 \%)$ of the mouth breathers showed labial action, $6(75 \%)$ showed mentalis action and $6(75 \%)$ showed tongue projection. In the electromyography evaluation, a higher muscular activity was verified, in the mouth breathers, when comparing to the nose breathers, in both lips, with a statistical significance. Conclusions: in this study it was verified that mouth breathers showed significant alterations in the swallowing functions when compared to the nose breathers that were observed through a clinical examination and electromyography evaluation.
\end{abstract}

KEYWORDS: Deglutition; Electromyography; Facial Muscles; Mouth Breathing; Stomatognathic System

\section{REFERÊNCIAS}

1. Berger WE. Allergic rhinitis in children: diagnosis and management strategies. Paediatr Drugs. 2004; 6(4):233-50.

2. Simas Netta ML, Maruo H, Vieira SR, Saga AY. Estudo cefalométrico comparativo das dimensões craniofaciais entre crianças respiradoras nasais e bucais, com maloclusão classe II, divisão 1. J Bras Ortod Ortop Facial. 2004; 9(49):41-7.

3. Lessa FCR, Enoki C, Feres MFN, Valera FCP, Lima WTA, Matsumoto MAN. Influência do padrão respiratório na morfologia craniofacial. Rev Bras Otorrinolaringol. 2005; 71(2):156-60.
4. Sollé D, Mello Júnior JF, Weckx LLM, Rosário Filho NA. II Consenso sobre rinites 2006. Rev Bras Alerg Imunopatol. 2006; 29(1):29-58.

5. Vianna-Lara MS, Caria PHF. Electromygraphic analysis of the upper lip in nose and mouth breathers. Braz J Oral Sci. 2006; 5(19):1203-8.

6. Frasson JMD, Magnani MBBA, Nouer DF, Siqueira VCV, Lunardi N. Estudo cefalométrico comparativo entre respiradores nasais e predominantemente bucais. Rev Bras Otorrinolaringol. 2006; 72(1):72-81.

7. Di Francesco RC, Passerotii G, Paulucci B, Miniti A. Respiração oral na criança: repercussões diferentes de acordo com o diagnóstico. Rev Bras Otorrinolaringol. 2004; 70(5):665-70. 
8. Menezes VA, Leal RB, Pessoa RS, Pontes RMS. Prevalência e fatores associados à respiração oral em escolares participantes do projeto Santo AmaroRecife, 2005. Rev Bras Otorrinolaringol. 2006; 72(3):394-9.

9. Lemos CM, Junqueira PAS, Goffi-Gomez MVS, Faria MEJ, Basso SC. Estudo da relação entre a oclusão dentária e a deglutição no respirador oral. Arq Int Otorrinolaringol. 2006; 10(2):114-8.

10. Mateu ME, Bertolotti MC, Schweiser $H$. Disgnacias como respuesta al desequilibrio funcional producido por hábitos de respiración bucal y deglución atípica. Rev Círc Argent Odontol. 2006; 63(199):26-31.

11. Cattoni DM, Fernandes FDM, Di Francesco RC, Latorre MRDO. Características do sistema estomatognático de crianças respiradoras orais: enfoque antroposcópico. Pró-Fono. 2007; 19(4):347-51.

12. Lemos CM, Wilhelmsen NSW, Mion OG, Mello Júnior JF. Alterações funcionais do sistema estomatognático em pacientes com rinite alérgica. Arq Int Otorrinolaringol. 2007; 11(4):380-6.

13. Coelho MF, Terra VHTC. Implicações clínicas em pacientes respiradores bucais. Rev Bras Patol Oral. 2004; 3(1):17-9.

14. Fraser $C$. Tongue thrust and its influence in orthodontics. Int J Orthod. 2006; 17(1):9-18.

15. Bianchini AP, Guedes ZCF, Vieira MM. Estudo da relação entre a respiração oral e o tipo facial. Rev Bras Otorrinolaringol. 2007; 73(4):500-5.

16. Marchesan IQ. Deglutição: diagnóstico e possibilidades terapêuticas. In: Marchesan IQ. Fundamentos em fonoaudiologia: aspectos clínicos da motricidade oral. Rio de Janeiro: Guanabara Koogan; 2005. p. 59-68.

17. Basmajian JV, De Luca CJ. Muscles Alive: their functions revelead by electromyography. 5. ed. Baltimore: Williams \& Wilkins; 1985.

18. Marchiori SC, Tomé MC. Estudo eletromiográfico dos músculos orbiculares superior e inferior da boca em crianças respiradoras nasais e bucais durante a emissão de sílabas. Pró-Fono. 1999; 11(1):1-7.

DOI: 10.1590/S1516-18462009005000046

RECEBIDO EM: 19/12/2008

ACEITO EM: 18/06/2009

Endereço para correspondência:

Tais Regina Hennig

Rua Marechal Floriano Peixoto, 1408 ap. 302

Santa Maria - RS

CEP: 97015-372

E-mail: tha_hennig@ hotmail.com
19. Tosello DO, Vitti M, Berzin F. EMG activity of the orbicularis oris and mentalis muscles in children with malocclusion, incompetent lips and atypical swallowing-part II. J Oral Rehabil. 1999; 26(8):644-9. 20. Dutra EH, Maruo H, Vianna-Lara MS. Electromyographic activity evaluation and comparison of the orbicularis oris (lower fascicle) and mentalis muscles in predominantly nose- or mouth-breathing subjects. Am J Orthod Dentofacial Orthop. 2006; 129(6):722.e1-722.e9.

21. De Luca CJ. The use of surface electromyography in biomechanics. J Appl Biomech. 1997; 13(2): 135-63.

22. Cram JR, Kasman GS, Holtz J. Introduction to surface electromyography. Maryland: Aspen Publishers; 1998.

23. Nagae M, Berzin F. Electromyography: applied in the phonoaudiology clinic. Braz J Oral Sci. 2004; 3(10):506-9.

24. Corrêa ECR, Bérzin F. Efficacy of physical therapy on cervical muscle activity and on body posture in school-age mouth breathing children. Int J Pediatr Otorhinolaryngol. 2007; 71(10):1527-35.

25. Cattoni DM. Alterações da mastigação e deglutição. In: Ferreira LP, Befi-Lopes DM, Limongi SCO. Tratado de fonoaudiologia. São Paulo: Roca; 2004. p. 277-91.

26. Tulley WJ. Methods of recording patterns of behavior of the oro-facial muscles using the electromyography. Dental Record. 1953; 73:741-8. 27. Silva AMT. Eletromiografia: avaliação dos músculos orbiculares orais da boa em crianças respiradoras bucais, pré e pós mioterapia [tese]. São Paulo (SP): Universidade Federal de São Paulo; 2000.

28. Tomé MC, Marchiori SC. Estudo eletromiográfico dos músculos orbiculares superior e inferior da boca em crianças respiradoras nasais e bucais durante o repouso com e sem contato labial. J Bras Ortodon Ortop Facial. 1998; 3(15):59-66.

29. Marchiori SC, Tomé MC. Análise eletromiográfica dos músculos orbiculares superior e inferior da boca em crianças respiradoras nasais e bucais durante a deglutição e o sopro. Rev Soc Bras Fonoaudiol. 1998; 2(4):16-21. 